\title{
Oscillations in critical shearing, application to fractures in glaciers
}

\author{
A. Pralong \\ Laboratory of Hydraulics, Hydrology and Glaciology, Swiss Federal Institute of Technology, 8092 Zürich, Switzerland
}

Received: 18 August 2006 - Revised: 23 November 2006 - Accepted: 24 November 2006 - Published: 12 December 2006

\begin{abstract}
Many evidences of oscillations accompanying the acceleration of critical systems have been reported. These oscillations are usually related to discrete scale invariance properties of the systems and exhibit a logarithmic periodicity. In this paper we propose another explanation for these oscillations in the case of shearing fracture. Using a continuum damage model, we show that oscillations emerge from the anisotropic properties of the cracks in the shearing fracture zone. These oscillations no longer exhibit a logarithmic but rather a power-law periodicity. The power-periodic oscillation is a more general formulation. Its reduces to a log-periodic oscillation when the exponent of the power-law equals one. We apply this model to fit the measured displacements of unstable ice masses of hanging glaciers for which data are available. Results show that power-periodic oscillations adequately fit the observations.
\end{abstract}

\section{Introduction}

Accelerating oscillations appear in a large variety of failure processes. They accompany the power-law acceleration observed prior to the failure and are usually described by logperiodic oscillations (see the review of Zhou and Sornette, 2002). Log-periodic oscillations appear in systems exhibiting discrete scale invariance properties (Sornette, 1998). In fracture of rocks, numerical simulations "indicate that the interplay between the heterogeneities of rock and the stress field generates dynamically such a scale invariance" (Sahimi and Arbabi, 1996) and log-periodic corrections to the powerlaw acceleration. Accelerating oscillations must be considered to perform an accurate prediction of the time of failure (Pralong et al., 2005).

Correspondence to: A. Pralong

(pralong@vaw.baug.ethz.ch)
We show in this paper that accelerating oscillations with another type of periodicity emerge in critical shearing processes because of the anisotropy of the orientation distribution of the cracks. These oscillations have a frequency proportional to a power of the time before failure and reduce to log-periodic oscillations when the exponent of the time before failure tends to one. To derive this power dependence we build a shearing fracture model. We do not consider each individual crack but the density of the cracks which develop in the shearing zone by using the theory of the continuum damage mechanics. Since individual cracks are not considered, discrete scale invariance properties of the crack pattern do not exist in the model and therefore do not play a role in the existence of these power-periodic oscillations. We apply this model to the displacement field prior to failure of unstable ice masses from hanging glaciers for which data are available. We therefore consider a viscoelastic, not necessarily Newtonian material, which describes the behavior of glacier ice.

The paper is organized in three parts. The first part introduces the assumptions of the shearing fracture model (Sect. 3), the differential equations and a numerical solution for the accelerating oscillations of the surface velocity (Sect. 4). The second part postulates further assumptions in order to derive an analytical solution (Sect. 5). Then the analytical solution is compared to measurements performed on two hanging glaciers (Sect. 6). The last part discusses the results (Sect. 7).

\section{Notations and definitions}

In what follows, vectors are denoted as bold, and second order tensors are underlined. We use symbolic and Cartesian tensor notation and employ the summation convention according to which summation is understood over doubly repeated indices.

Published by Copernicus GmbH on behalf of the European Geosciences Union and the American Geophysical Union. 
Let $\boldsymbol{x}=\tilde{\boldsymbol{x}}(t, \boldsymbol{X})$ represent the position of the material point of the body in the present (i.e., time $t$ ) configuration whilst $\boldsymbol{X}$ is its position in the corresponding reference configuration. Let $\boldsymbol{v}(t, \boldsymbol{x})=(\partial \tilde{\boldsymbol{x}} / \partial t)(t, \boldsymbol{X}(t, \boldsymbol{x}))$ be the spatial velocity vector, $\underline{L}(t, \boldsymbol{x})=(\partial \boldsymbol{v} / \partial \tilde{\boldsymbol{x}})(t, \boldsymbol{x}, \boldsymbol{X}(t, \boldsymbol{x}))$ the velocity gradient tensor, $\underline{\dot{\epsilon}}=\frac{1}{2}\left(\underline{L}+\underline{L}^{T}\right)$ the stretching or strain rate tensor and $\underline{W}=\frac{1}{2}\left(\underline{L}-\underline{L}^{T}\right)$ the spin tensor. The dyadic products of two vectors $\boldsymbol{a}, \boldsymbol{b}$ will be denoted by $\boldsymbol{a} \otimes \boldsymbol{b}$. Moreover, the Lie [,] and Jacobi $\langle$,$\rangle brackets of two second$ rank tensors $\underline{A}, \underline{B}$ are defined by $[\underline{A}, \underline{B}]=\underline{A} \underline{B}-\underline{B} \underline{A}$ and $\langle\underline{A}, \underline{B}\rangle=\underline{A} \underline{B}+\underline{B} \underline{A}$, respectively. The first and second invariants of a second rank tensor $\underline{A}$ are defined as $I_{\underline{A}}=\operatorname{Tr} \underline{A}$ and $I_{\underline{A}}=\frac{1}{2}\left((\operatorname{Tr} \underline{A})^{2}-\operatorname{Tr}\left(\underline{A}^{2}\right)\right)$, respectively.

\section{Background and assumptions}

The processes of the glacier ice are modeled in the Eulerian description. The temperature in the glacier is assumed to be constant and the ice is cold (temperature below the melting point). The heat balance equation is therefore not considered.

The ice and the cracks are modeled as a unique medium, according to the theory of the continuum damage mechanics. We assume that the opening of the cracks which develop in the damaged ice (ice plus cracks) is negligible, since the mean deformation is shearing ${ }^{1}$. Because the virgin ice (ice without cracks) is incompressible and the opening of the cracks is ignored, the damaged ice is thus volume preserving, so that the equation of mass conservation reads

$v_{i}, i=0$,

in which $v_{i}$ is the velocity of the damaged ice.

The acceleration terms in the balance of linear momentum are neglected because of the slow creeping character of the ice motion. This so-called Stokes assumption is commonly adopted in glaciology. The linear momentum equations then read

$\sigma_{i j},{ }_{j}=-\rho b_{i}$,

in which $\sigma_{i j}, \rho, b_{i}$ are the Cauchy stress tensor, the density and the body force vector of the damaged ice, respectively.

A variable $\underline{D}$ (damage variable) is introduced to quantify the amount of cracks in the ice, as well as the anisotropy of the orientation distribution of the cracks. We restrict ourself here to an orthotropic description of the anisotropy, so that the damage variable is a symmetric second rank tensor. In that case, and since the ice is cold, the balance equation for $\underline{D}$ is described by (Pralong et al., 2006)

$\underline{\dot{D}}+[\underline{D}, \underline{W}]=\underline{f}$.

\footnotetext{
${ }^{1}$ We assume later on uniformity of the processes in the flow direction (see Eq. 13), which leads to a pure shearing flow. Possible tensile cracks at the glacier surface are not relevant here, since the critical damage accumulates at the glacier base due to shearing.
}

The left-hand side is the Jaumann derivative (a spatially objective derivative) with the material derivative given by

$\underline{\dot{D}}=\frac{\partial \underline{D}}{\partial t}+\operatorname{grad} \underline{D} \cdot \boldsymbol{v}$.

The spin tensor $\underline{W}$ is defined in Sect. 2. $f$ is the source term of damage (called the dynamic function of damage), which describes the increase (cracking) and decrease (healing) of damage with time.

\subsection{Damage evolution}

In the dynamic function of damage, healing is neglected, since the ice temperature is substantially below melting. Cracking is modeled with a Kachanow type law valid for orthotropic damage (Kachanov, 1999; Rabotnov, 1969; Murakami and Ohno, 1980). So, in nearly plane flow $\underline{f}$ reads

$$
\begin{aligned}
\underline{f}= & B\left(\sigma_{x z}-\sigma_{\mathrm{th}}\right)^{r}\left(1-\frac{D_{x x}+D_{z z}}{2}\right)^{-k} \\
& {\left[(1-\gamma) \underline{I}+\gamma \boldsymbol{v}^{(1)} \otimes \boldsymbol{v}^{(1)}\right], }
\end{aligned}
$$

where $B, r$ and $k$ are evolution parameters, $\sigma_{\text {th }}$ is the stress threshold accounting for damage nucleation and $\underline{I}$ is the second order unit tensor whilst $\sigma_{x z}$ is assumed to describe the influence of the stress state on the damage evolution, since the ice motion is close to a pure shearing motion (see relation 13$)^{2}$. The expression $1-\frac{D_{x x}+D_{z z}}{2}$ expresses the positive feedback of the damage on its evolution ${ }^{3}$. The orientation of damage growth (last factor on the right hand side of Eq. 5) is expressed as a linear combination of isotropic $(\gamma=0)$ and orthotropic $(\gamma=1)$ contributions. The orthotropic orientation is modeled by the dyadic product $\boldsymbol{v}^{(1)} \otimes \boldsymbol{v}^{(1)}$, where $\boldsymbol{v}^{(1)}$ is the unit eigenvector associated with the maximum eigenvalue ${ }^{4}$ of the effective stress tensor $\underline{\tilde{\sigma}}^{5}$, which is defined as (Pralong et al., 2006)

$\underline{\tilde{\sigma}}=\frac{1}{2}\left\langle(\underline{I}-\underline{D})^{-1}, \underline{\sigma}\right\rangle$.

\subsection{Stress of the damaged ice}

Since the glacier motion is observed over a long period, the elastic deformations are assumed to be negligible compared to the viscous deformations. The viscous stress (nonequilibrium stress) thus equals the global stress $\underline{\sigma}$. The

\footnotetext{
${ }^{2} \mathrm{~A}$ more complex description of the influence of the stress state accounting for invariants of the stress tensor (Pralong et al., 2006) does not change the behavior of the damage evolution and the shape of the accelerating oscillations.

${ }^{3}$ It will appear later on that the term $D_{x x}+D_{z z}$ is proportional to the first invariant of the damage tensor $\underline{D}$. For $\gamma=1$ (full anisotropy), $D_{x x}+D_{z z}$ equals the first invariant since $D_{y y}=0$.

${ }^{4}$ This can be a positive or negative value; that is, the anisotropy can develop in tension and compression regimes.

${ }^{5}$ The effective stress corresponds to the stress redistributed because of the presence of cracks.
} 
viscous stress can be derived from a dissipation potential $\varphi(\underline{\dot{\epsilon}}, \underline{I}-\underline{D}$ ), which depends on the strain rate tensor $\underline{\dot{\epsilon}}$ (defined in Sect. 2) and the damage tensor $\underline{D}$. According to the assumption of incompressibility, the isotropic expansion of the stress leads to (Pralong et al., 2006)

$$
\begin{aligned}
\underline{\sigma}^{\prime}= & \frac{\partial \varphi(\underline{\dot{\epsilon}}, \underline{I}-\underline{D})}{\partial \dot{\hat{\epsilon}}} \\
= & -\delta_{12} \underline{\dot{\dot{\epsilon}}}+2\left(\delta_{21}+\delta_{22} I_{\langle\underline{I}-\underline{D}, \underline{\dot{\epsilon}}\rangle}\right)(\underline{I}-\underline{D})^{\prime} \\
& +4\left(\delta_{31}+\delta_{32} I_{\left\langle\underline{I}-\underline{D}, \underline{\dot{\epsilon}^{2}}\right\rangle}\right) \underline{\tilde{\dot{\epsilon}}}^{\prime}+\ldots
\end{aligned}
$$

where higher order terms have been neglected. The prime denotes the deviatoric part of the tensor. The quantities $\delta_{i j}$ and the effective strain rate tensor $\tilde{\tilde{\epsilon}}$ are defined as

$$
\begin{aligned}
& \delta_{12}=\frac{\partial \varphi}{\partial I_{\underline{\dot{\epsilon}}}}, \quad \delta_{21}=\frac{\partial \varphi}{\partial I_{\langle\underline{I}-\underline{D}, \dot{\underline{\epsilon}}\rangle}}, \delta_{22}=\frac{\partial \varphi}{\partial I_{\langle\underline{I}-\underline{D}, \underline{\dot{\epsilon}}\rangle}}, \\
& \delta_{31}=\frac{\partial \varphi}{\partial I_{\left\langle\underline{I}-\underline{D}, \dot{\epsilon}^{2}\right\rangle}}, \quad \delta_{32}=\frac{\partial \varphi}{\partial I_{\left\langle\underline{I}-\underline{D}, \underline{\dot{\epsilon}}^{2}\right\rangle}}, \\
& \underline{\tilde{\epsilon}}=\frac{1}{2}\langle\underline{I}-\underline{D}, \underline{\dot{\epsilon}}\rangle .
\end{aligned}
$$

The terms $\delta_{12}$ and $2\left(\delta_{21}+\delta_{22} I_{\langle I-D, \dot{\epsilon}\rangle}\right)$ have been neglected in the work by Pralong et al. (2006), such that the effective strain rate $\tilde{\tilde{\epsilon}}$ becomes identical to the effective strain rate emerging from the postulation of the stress equivalence principle, which is commonly adopted in the theory of the continuum damage mechanics (Lemaitre, 1996). The factor $4\left(\delta_{31}+\delta_{32} I_{\left\langle\underline{I}-D, \dot{\epsilon}^{2}\right\rangle}\right)$ is the viscosity of the damaged ice. It will be denoted $\eta_{\dot{\epsilon}}$ and is assumed to equal

$\eta_{\dot{\epsilon}}=A^{-\frac{1}{n}}\left(-I_{\underline{\dot{\epsilon}}}\right)^{\frac{1-n}{2 n}}$,

which corresponds to Glen's viscosity (Glen, 1952). $A$ is the fluidity parameter and $n$ the power-law exponent.

In this paper, we do not neglect the term $2\left(\delta_{21}+\delta_{22} I_{\langle\underline{I}-\underline{D}, \dot{\epsilon}\rangle}\right)$ of the isotropic expansion (7), which is a pure damage contribution to the stress. This factor plays the role of a damage viscosity. It is assumed to be constant and will be denoted $\eta_{D}$. By considering the relation $\underline{\sigma}^{\prime}=\underline{\sigma}+p \underline{I}$, the Cauchy stress tensor reads

$\underline{\sigma}=-p \underline{I}+\eta_{D}(\underline{I}-\underline{D})^{\prime}+\eta_{\dot{\epsilon}} \tilde{\dot{\epsilon}}^{\prime}$,

with $p$ the pressure. For virgin ice $(\underline{D}=\underline{0})$ relation (11) reduces to the classical Glen flow law (Glen, 1952). The reason to consider $\eta_{D}$ will appear later on.

\subsection{Geometrical assumptions}

Henceforth, neglecting boundary effects from the glacier margins, we assume strictly plane strain flow (in the plane $x-z)$, i.e.

$\frac{\partial}{\partial y}=0 \quad$ and $\quad v_{y}=0$,

and uniformity of the processes in the flow direction as in a parallel sided slab flow, i.e.

$\frac{\partial}{\partial x}=0$.

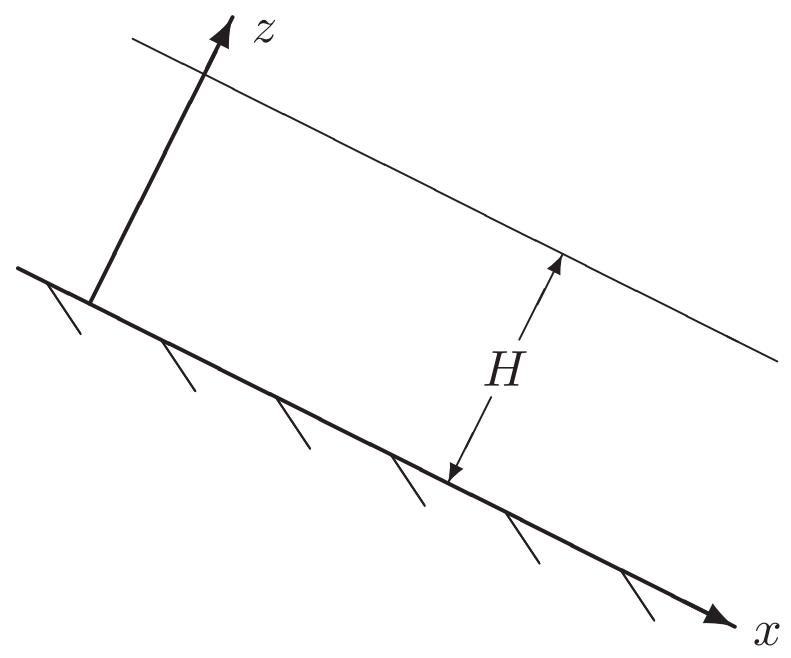

Fig. 1. Geometry of the glacier, as used for the numerical and analytical models.

The geometry of the glacier is shown in Fig. 1.

We finally suppose that damage anisotropy does not develop in the y-direction. Therefore, the first eigenvector $\boldsymbol{v}^{(1)}$ in Eq. (5) is imposed to have the property

$\boldsymbol{v}^{(1)} \cdot \boldsymbol{y}=0$.

We further assume ${ }^{6}$

$D_{x y}=D_{y z}=0$.

The boundary condition at the glacier base is no-slip since the glacier is frozen to its bed. It reads

$\left.\boldsymbol{v}\right|_{z=0}=\mathbf{0}$,

and the glacier surface is traction free

$\left.\underline{\sigma}\right|_{z=H} \cdot \boldsymbol{n}=\mathbf{0}$,

where $\boldsymbol{n}$ is the vector normal to the surface and $H$ is the glacier thickness.

\subsection{Localization of damage}

On the basis of empirical observations and numerical modeling we assume that damage localizes close to the glacier bed, i.e., near $z=0$. This localization is due to the fact that damage cannot evolve below the stress threshold $\sigma_{\text {th }}$ for damage accumulation; that is, it develops first at the glacier base when the glacier reaches a critical thickness, i.e., when the basal stress reaches the stress threshold $\sigma_{\text {th }}$ (Pralong and Funk, 2005). Moreover, the thickening of the glacier due to snow

\footnotetext{
${ }^{6}$ This is equivalent to postulate $D_{x y}\left(t=t_{0}\right)=D_{y z}\left(t=t_{0}\right)=0$ where $t_{0}$ is the time corresponding to the beginning of the failure process. This can be demonstrated with the help of Eqs. (3), (5), (12), (13), (14) and (19).
} 


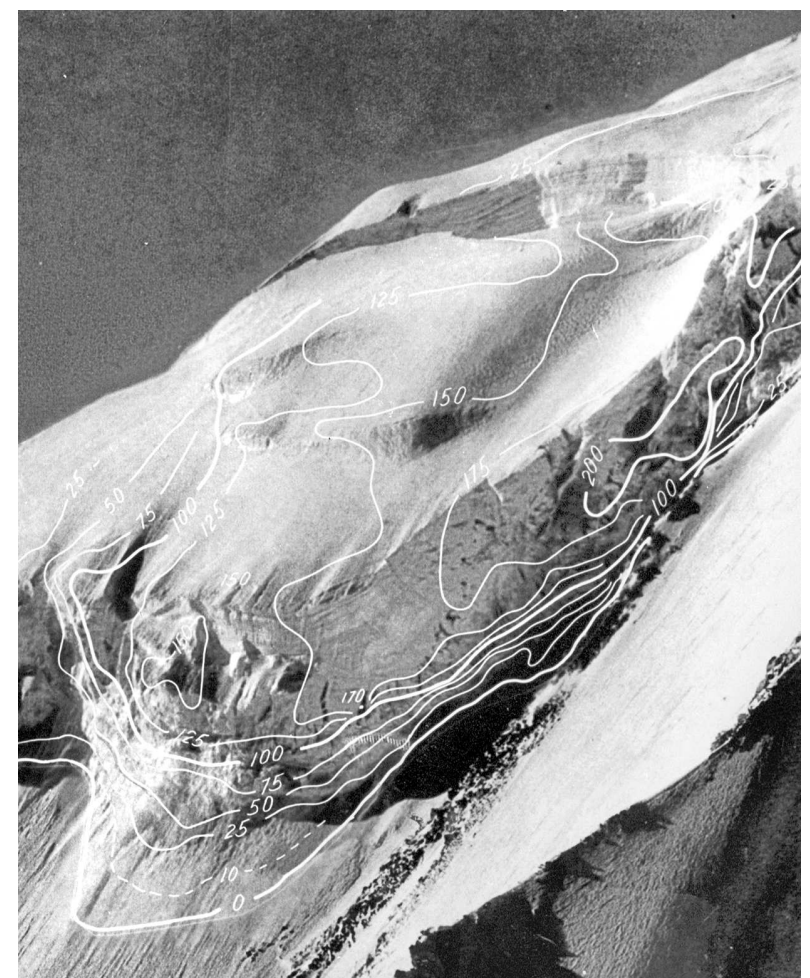

Fig. 2. Surface displacement (cm) of an unstable ice chunk in 18 days, 10 months prior to the 1973 failure on the Weisshorn east face in the Valais Alps (Switzerland). Picture by A. Flotron, 1972 (detail); see also Röthlisberger (1981).

accumulation is much slower than the processes of damage evolution. It implies that the active layer, where damage accumulates, has no time to significantly thicken during the failure process. Figure 2 shows the measured surface velocity distribution of an unstable ice mass corresponding to the geometrical assumptions introduced above. The velocity gradient along the z-direction is high at the base due to the localization of damage. This behavior has been reproduced numerically by Pralong and Funk (2006).

We postulate that the active layer, where damage develops, has constant thickness $\Delta H$, which is small compared to the glacier thickness and can be neglected, i.e.

$$
\Delta H \approx 0
$$

This relation shows that the damage layer in the model is extremely thin and located at $z=0$. The damage process can thus be understood to act as a boundary condition for the glacier, in the same way as the effect of sedimentary layers which are concentrated at the base. Above this layer damage accumulation is ignored.

\section{Equations for damage evolution and glacier velocity}

\subsection{Derivation and equations}

The integration of the mass balance equation (1) with assumptions $\left(12_{a}\right)$ and (13) and condition (16) leads to

$v_{z} \equiv 0$.

By integrating the balance equations of linear momentum (Eq. 2 for $i=x, z$ ) over the thickness of the glacier, one obtains, with the help of assumptions $\left(12_{a}\right)$ and (13) and condition (17)

$\sigma_{x z}=\rho b_{x}(H-z)$,

$\sigma_{z z}=\rho b_{z}(H-z)$.

With assumptions (12) and (13) and Eq. (19) the strain rate tensor $\dot{\epsilon}$ reduces to

$\underline{\dot{\epsilon}}=\frac{1}{2} \frac{\partial v_{x}}{\partial z}\left[\begin{array}{lll}0 & 0 & 1 \\ 0 & 0 & 0 \\ 1 & 0 & 0\end{array}\right]$,

and using this result, the stress tensor $\underline{\sigma}$ (Eq. 11) with relation (15) takes the form

$$
\begin{aligned}
& \sigma_{x x}=-p+\frac{1}{3} \eta_{D}\left(-2 D_{x x}+D_{y y}+D_{z z}\right)-\frac{1}{6} \eta_{\dot{\epsilon}} \frac{\partial v_{x}}{\partial z} D_{x z}, \\
& \sigma_{y y}=-p+\frac{1}{3} \eta_{D}\left(D_{x x}-2 D_{y y}+D_{z z}\right)+\frac{1}{3} \eta_{\dot{\epsilon}} \frac{\partial v_{x}}{\partial z} D_{x z}, \\
& \sigma_{z z}=-p+\frac{1}{3} \eta_{D}\left(D_{x x}+D_{y y}-2 D_{z z}\right)-\frac{1}{6} \eta_{\dot{\epsilon}} \frac{\partial v_{x}}{\partial z} D_{x z}, \\
& \sigma_{x z}=-\eta_{D} D_{x z}+\frac{1}{2} \eta_{\dot{\epsilon}} \frac{\partial v_{x}}{\partial z}\left(1-\frac{D_{x x}+D_{z z}}{2}\right) .
\end{aligned}
$$

Moreover, with Eq. $\left(20_{a}\right)$ the equations for $\sigma_{z z}$ and $\sigma_{x z}$ furnish relations for the pressure $p$ and the velocity $v_{x}$ in the $x$-direction as follows

$p=\frac{1}{3} \eta_{D}\left(D_{x x}+D_{y y}-2 D_{z z}\right)-\rho b_{z}(H-z)-\frac{1}{3} C D_{x z}$

$\eta_{\dot{\epsilon}} \frac{\partial v_{x}}{\partial z}=2 C$,

where

$C=\frac{\rho b_{x}(H-z)+\eta_{D} D_{x z}}{1-\frac{D_{x x}+D_{z z}}{2}}$.

With Eqs. $\left(20_{b}\right),(22),(23)$ and (24), the stress tensor $\underline{\sigma}$ can be rewritten as

$$
\begin{aligned}
& \sigma_{x x}=\rho b_{z}(H-z)+\eta_{D}\left(D_{z z}-D_{x x}\right), \\
& \sigma_{y y}=\rho b_{z}(H-z)+\eta_{D}\left(D_{z z}-D_{y y}\right)+C D_{x z}, \\
& \sigma_{z z}=\rho b_{z}(H-z), \\
& \sigma_{x z}=\rho b_{x}(H-z),
\end{aligned}
$$

and with Eqs. (10) and (21) the differential equation (24) for the velocity becomes

$$
\frac{\partial v_{x}}{\partial z}=2 A C^{n} .
$$


Imposing the assumption (16) and the approximation (18), the integration of the velocity along the glacier thickness leads to

$$
\begin{aligned}
v_{x}(z)= & 2 A\left(\left.C\right|_{z=0}\right)^{n} \Delta H+2 \frac{A}{n+1}\left(\rho b_{x}\right)^{n} \\
& \left(H^{n+1}-(H-z)^{n+1}\right) .
\end{aligned}
$$

The first term of Eq. (28) describes contribution to the velocity due to the damage active layer and the second term gives the shearing contribution to the velocity profile in the glacier. The first term depends on time, since $C$ depends on damage $\underline{D}$, which is a function of time. The second term is time independent. We have to propose in this equation a value for $\Delta H$ (which is not infinitely small, otherwise the velocity of the active layer would vanish). $2 A \Delta H$ can also be considered as a global parameter describing the viscosity of the basal layer ${ }^{7}$.

To compute the evolution of the velocity $v_{x}$, we can simplify the balance equation of damage (3). Since, with assumptions (12) and (13) and Eq. (19), the spin tensor takes the form

$\underline{W}=\frac{1}{2} \frac{\partial v_{x}}{\partial z}\left[\begin{array}{ccc}0 & 0 & 1 \\ 0 & 0 & 0 \\ -1 & 0 & 0\end{array}\right]$,

use of Eqs. (18), (25) and (27) allows the balance equations of damage for the active layer to be written as

$$
\begin{array}{ll}
\frac{\partial D_{x x}}{\partial t}=2 A\left(\frac{\rho b_{x} H+\eta_{D} D_{x z}}{1-\frac{D_{x x}+D_{z z}}{2}}\right)^{n} D_{x z} & +f_{x x}\left(\underline{D},\left.\underline{\sigma}\right|_{z=0}\right), \\
\frac{\partial D_{y y}}{\partial t}= & f_{y y}\left(\underline{D},\left.\underline{\sigma}\right|_{z=0}\right) \\
\frac{\partial D_{z z}}{\partial t}=-2 A\left(\frac{\rho b_{x} H+\eta_{D} D_{x z}}{1-\frac{D_{x x}+D_{z z}}{2}}\right)^{n} D_{x z} & +f_{z z}\left(\underline{D},\left.\underline{\sigma}\right|_{z=0}\right), \\
\frac{\partial D_{x z}}{\partial t}=A\left(\frac{\rho b_{x} H+\eta_{D} D_{x z}}{1-\frac{D_{z z}+D_{x x}}{2}}\right)^{n}\left(D_{z z}-D_{x x}\right)+f_{x z}\left(\underline{D},\left.\underline{\sigma}\right|_{z=0}\right),
\end{array}
$$

where $f_{i j}$ are the components of the dynamic function of damage (5). The stress tensor $\underline{\sigma}$ used to evaluate $\underline{f}$ is given by relation (26).

With Eq. $\left(26_{d}\right)$ and the relation $\sigma_{\mathrm{th}}=\sigma_{x z}(z=\Delta H)$, one can write

$$
\frac{\sigma_{\mathrm{th}}}{\sigma_{x z}}=\frac{H-\Delta H}{H} \text {. }
$$

The term $\sigma_{x z}-\sigma_{\text {th }}$ in the damage evolution law (5) can thus be expressed as

$\sigma_{x z}-\sigma_{\text {th }}=\sigma_{x z} \frac{\Delta H}{H}$.

As for Eq. (28) a physical value for $\Delta H$ must be proposed, otherwise no damage would develop in the active layer. According to Eq. (5) a global parameter $B\left(\frac{\Delta H}{H}\right)^{r}$ describing the rate of damage evolution for the damage layer can be proposed for the dynamic function of damage (5).

\footnotetext{
${ }^{7}$ in a similar way as the parameter $R$ in the constitutive relation $v_{x}=R \sigma_{x z}^{m}$ for basal sedimentary layers, where $m$ is the power-law exponent and $R$ is the fluidity parameter for the layer.
}

Table 1. Values of the parameters used in the model. Parameters of the damage evolution are given by Pralong and Funk (2005). Note that $k=0$ in Pralong and Funk (2005), but here $k=r$ since the dynamic function of damage (5) depends on stress and not on effective stress. Parameters of the ice flow law are given by Paterson (1994).

$\begin{array}{llll}A & =5 \times 10^{-24}\left(\mathrm{~Pa}^{-n} \mathrm{~s}^{-1}\right) & k & =0.43 \\ B & =1.7 \times 10^{-9}\left(\mathrm{~Pa}^{-r} \mathrm{~s}^{-1}\right) & n & =3 \\ b_{x} & =9.81 \sin \left(45^{\circ}\right)\left(\mathrm{m} \mathrm{s}^{-2}\right) & r & =0.43 \\ b_{z} & =9.81 \cos \left(45^{\circ}\right)\left(\mathrm{m} \mathrm{s}^{-2}\right) & \gamma & =1 \\ D_{\mathrm{c}} & =0.9 & \Delta H & =0.01 H(m) \\ H & =30(m) & \eta_{D} & =0.1 \sigma_{x z}(z=0)(\mathrm{Pa}) \\ & & \rho & =910\left(\mathrm{~kg} \mathrm{~m}^{-3}\right)\end{array}$

\subsection{Example}

In order to present the effect on the ice flow of the damage induced anisotropy, we shall solve the pertinent equations above to find the evolution of the glacier velocity and the damage in the basal layer. The values of the model parameters are summarized in Table 1. The glacier dimensions correspond approximatively to the Weisshorn hanging glacier as observed in 2005 (see Sect. 6).

Since we want to analyze the effect of the anisotropy, we set $\gamma=1$. The value of the damage viscosity $\eta_{D}$ influences the amplitude of the oscillations accompanying the global trend (see Fig. 4c) and is so set to obtain amplitudes comparable to those observed on glaciers. The fracture is assumed to happen when the velocity is finite (before the finite time singularity of the velocity occurs, see Eq. 33) as is usually postulated in failure models. Fracture happens when the component $D_{z z}$ of the damage tensor reaches a critical damage threshold $D_{\text {c }}$ accounting for the transition from subcritical to critical crack growth (Lemaitre, 1996); that is, when a macroscopic fracture propagates in the basal layer parallel to the glacier base. Unfortunately, the value of the damage threshold $D_{\mathrm{c}}$ has not been determined for shearing; in this example it is arbitrarily set equal to $D_{\mathrm{c}}=0.9^{8}$.

Figure $3 \mathrm{a}$ presents the simulation of the acceleration of the glacier. The acceleration (without oscillations) can be described by a power-law equation (e.g. Voight, 1988)

$v_{x}(t, z=H)=v_{x 0}+a_{\Omega}\left(t_{\mathrm{f}}-t\right)^{-m_{\Omega}}$,

where $a_{\Omega}$ and $m_{\Omega}$ are the parameters characterizing the acceleration and $v_{x 0}$ is a constant velocity. The model velocity at the glacier surface $v_{x}(z=H)$ is fitted with function (33) (Fig. 3a). The residuals of the fit show oscillations with a frequency increasing with time (Fig. 3b). Figure 3c shows the evolution of the damage tensor components. According

\footnotetext{
${ }^{8}$ This value influences the duration of the subcritical cracking process and the number of oscillations but does neither modify their frequency and amplitude nor the power-law acceleration.
} 

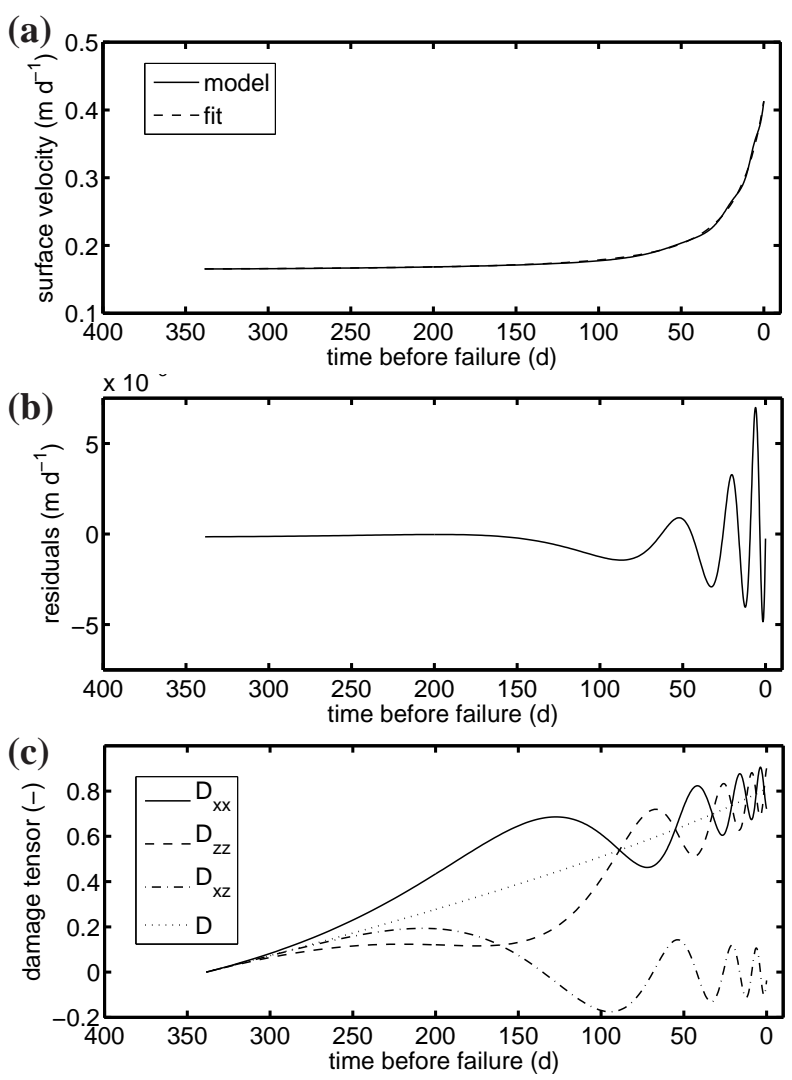

Fig. 3. Simulation of the failure of the hanging glacier with model parameters given by Table 1 . (a) Surface velocity $v_{x}(t, z=H)$ versus time before failure. (b) Residuals of the surface velocity fitted with Eq. (33). (c) Evolution of the components of $\underline{D} . D$ is defined in Eq. (42). Note that $D_{y y}=0$ since $\gamma=1$.

to Eqs. (25) and (28), the term $D=\frac{1}{2}\left(D_{x x}+D_{z z}\right)$ governs the global acceleration. Note that $D$ does not oscillate, whilst $D_{x x}$ and $D_{z z}$ do. The component $D_{x z}$ induces the oscillations of the surface velocity. The reason for the oscillations of $D_{x z}$ will be discussed in Sects. 5 and 7.

The shape of the oscillations depends on the choice of the parameter values (Table 1). These values (at least those for the damage evolution) are subject to uncertainties (Pralong and Funk, 2005). Moreover, many combinations of parameters lead to oscillations (see the analytical solution below, Sect. 5.4 and Appendix A). Therefore, this example has not the purpose to describe the natural event precisely, but to show that oscillations may emerge in a model of anisotropic damage evolution for shearing fractures.

\section{Analysis of the oscillations}

\subsection{Assumptions}

In order to find an analytical solution, we introduce three further assumptions.
We first neglect the influence of the damage on the orientation of the damage evolution; that is, the first eigenvector $\boldsymbol{v}^{(1)}$ in relation (5) is replaced by the first eigenvector of the stress tensor for virgin ice. With Eqs. (14) and (26), the tensor of the damage-evolution orientation with no damage dependency $\left.\boldsymbol{v}^{(1)} \otimes \boldsymbol{v}^{(1)}\right|_{\underline{D}=\underline{0}}$ reads

$\left.\boldsymbol{v}^{(1)} \otimes \boldsymbol{v}^{(1)}\right|_{\underline{D}=\underline{0}}=\frac{1}{2}\left[\begin{array}{lll}1 & 0 & 1 \\ 0 & 0 & 0 \\ 1 & 0 & 1\end{array}\right]$.

Figure 4a shows the values of the components of $\boldsymbol{v}^{(1)} \otimes \boldsymbol{v}^{(1)}$. They are oscillating around the values of relation (34). Figure $4 \mathrm{~b}$ shows the impact of this assumption on the oscillations of the surface velocity using the numerical example of Sect. 4.2. The amplitude of the oscillations is affected by the assumption. On the other hand, the frequency of the oscillations and the global acceleration of the glacier are not modified $^{9}$.

For the second assumption, we now introduce the amplitude of the oscillations to be small compared to the global velocity. With Eqs. (25) and (28), this assumption reads

$\rho b_{x} H+\eta_{D} D_{x z} \approx \rho b_{x} H$,

and can be expressed with Eq. $\left(26_{d}\right)$ and since $\left|D_{x z}\right|<1$ as

$\sigma_{x z}(z=0) \gg \eta_{D}$.

Figure $4 c$ compares the oscillations at the glacier surface with different values of $\eta_{D}$ using the numerical example of Sect. 4.2. The amplitude of the oscillations increases with increasing $\eta_{D}$. On the other hand, the frequency and the global acceleration do not vary.

Third, we postulated the failure to occur at infinite velocity; that is, according to Eqs. (25) and (28), when the damage $D=\frac{D_{x x}+D_{z z}}{2}$ reaches the critical damage $D_{\mathrm{c}}=1$. The hypothesis reads

$D_{\mathrm{c}}=1=D\left(t=t_{\mathrm{f}}\right)$

\subsection{Dimensionless representation}

The dimensionless description of the equations is based on the three following normative quantities

$\sigma_{x z}(z=0), \quad H \quad$ and $\quad v_{x}(\underline{D}=\underline{0}, z=H)$,

which are the basal shear stress, the glacier thickness and the surface velocity of the glacier before damage appears, respectively. The dimensionless quantities (overlined in

\footnotetext{
${ }^{9}$ The global acceleration is not modified since $\left(\boldsymbol{v}^{(1)} \otimes \boldsymbol{v}^{(1)}\right)_{x x}+\left(\boldsymbol{v}^{(1)} \otimes \boldsymbol{v}^{(1)}\right)_{z z}=\left.\left(\boldsymbol{v}^{(1)} \otimes \boldsymbol{v}^{(1)}\right)_{x x}\right|_{D=0}$ $+\left.\left(\boldsymbol{v}^{(1)} \otimes \boldsymbol{v}^{(1)}\right)_{z z}\right|_{\underline{D}=\underline{0}}$
} 
Eq. 39) used in the following developments are (with the help of Eqs. 5, 26 and 28)

$\begin{array}{llll}\bar{D} & =\underline{D}, & \bar{A}=\frac{n+1}{2}, & \overline{\Delta H}=\frac{\Delta H}{H}, \\ \bar{B}=B \frac{H \sigma_{x z}^{r}(z=0)}{v_{x}(\underline{D}=\underline{0}, z=H)}, & \overline{\eta_{D}}=\frac{\eta_{D}}{\sigma_{x z}(z=0)}, & \overline{\rho b_{x} H}=\bar{\sigma}_{x z}(\bar{z}=0) \stackrel{(39)}{=1 .}\end{array}$

In the rest of Sect. 5 all equations are described in the dimensionless representation and the overlines are omitted.

\subsection{Derivation}

With Eqs. (5), (32), (34), (35) and (39), the balance equation of damage (30), expressed in dimensionless form, becomes

$$
\begin{array}{lll}
\frac{\partial D_{x x}}{\partial t}=2 A\left(1-\frac{D_{x x}+D_{z z}}{2}\right)^{-n} D_{x z} & + & \left(1-\frac{\gamma}{2}\right) B \alpha\left(1-\frac{D_{x x}+D_{z z}}{2}\right)^{-k}, \\
\frac{\partial D_{y y}}{\partial t}= & & (1-\gamma) B \alpha\left(1-\frac{D_{x x}+D_{z z}}{2}\right)^{-k}, \\
\frac{\partial D_{z z}}{\partial t}=-2 A\left(1-\frac{D_{x x}+D_{z z}}{2}\right)^{-n} D_{x z} & +\left(1-\frac{\gamma}{2}\right) B \alpha\left(1-\frac{D_{x x}+D_{z z}}{2}\right)^{-k}, \\
\frac{\partial D_{x z}}{\partial t}= & A\left(1-\frac{D_{x x}+D_{z z}}{2}\right)^{-n}\left(D_{z z}-D_{x x}\right)+\frac{\gamma}{2} B \alpha\left(1-\frac{D_{x x}+D_{z z}}{2}\right)^{-k},
\end{array}
$$

where

$\alpha=\left(\frac{\Delta H}{H}\right)^{r}$.

Considering the parameter transformation

$D=\frac{D_{x x}+D_{z z}}{2}$ and $D^{-}=\frac{D_{z z}-D_{x x}}{2}$,

the balance of damage (40) becomes

$$
\begin{aligned}
& \frac{\partial D}{\partial t}=\left(1-\frac{\gamma}{2}\right) B \alpha(1-D)^{-k}, \\
& \frac{\partial D_{y y}}{\partial t}=(1-\gamma) B \alpha(1-D)^{-k}, \\
& \frac{\partial D^{-}}{\partial t}=-2 A(1-D)^{-n} D_{x z}, \\
& \frac{\partial D_{x z}}{\partial t}=2 A(1-D)^{-n} D^{-}+\frac{\gamma}{2} B \alpha(1-D)^{-k} .
\end{aligned}
$$

When integrating Eq. $\left(43_{a}\right)$, one obtains

$$
1-D=\left(\left(1-\frac{\gamma}{2}\right)(k+1) B \alpha\right)^{\frac{1}{k+1}}\left(t_{\mathrm{f}}-t\right)^{\frac{1}{k+1}},
$$

in which $t_{\mathrm{f}}$ is the time of failure. Combining Eqs. $\left(43_{c}\right)$ and $\left(43_{d}\right)$ and considering Eq. (44) we obtain an ordinary differential equation for $D_{x z}$

$$
\begin{aligned}
& \frac{1}{4} \frac{1}{A^{2}}\left(\left(1-\frac{\gamma}{2}\right)(k+1) B \alpha\right)^{\frac{2 n}{k+1}}\left(t_{\mathrm{f}}-t\right)^{\frac{2 n}{k+1} \frac{\partial^{2} D_{x z}}{\partial t^{2}}} \\
& -\frac{1}{4} \frac{1}{A^{2}} \frac{n}{k+1}\left(\left(1-\frac{\gamma}{2}\right)(k+1) B \alpha\right)^{\frac{2 n}{k+1}}\left(t_{\mathrm{f}}-t\right)^{\frac{2 n}{k+1}-1} \frac{\partial D_{x z}}{\partial t}+D_{x z} \\
& =\frac{\gamma}{8} \frac{B}{A^{2}} \alpha\left(\left(1-\frac{\gamma}{2}\right)(k+1) B \alpha\right)^{\frac{2 n-k}{k+1}}\left(t_{\mathrm{f}}-t\right)^{\frac{2 n-2 k-1}{k+1}}\left(\frac{k-n}{k+1}\right) .
\end{aligned}
$$

This linear second order ODE describes the oscillations of $D_{x z}$.

With Eqs. (35) and (39), expression $\left(1+\eta_{D} D_{x z}\right)^{n}$ can be approximated as

$$
\left(1+\eta_{D} D_{x z}\right)^{n} \approx 1+n \eta_{D} D_{x z} .
$$

With Eqs. (25), (38), (39), (42a), (44) and (46) the Eq. (28) of the surface velocity of the glacier becomes

$$
v_{x}(t, z=1)=v_{x 0}+a_{\Omega}\left(t_{\mathrm{f}}-t\right)^{-m_{\Omega}}\left(1+n \eta_{D} D_{x z}\right) .
$$
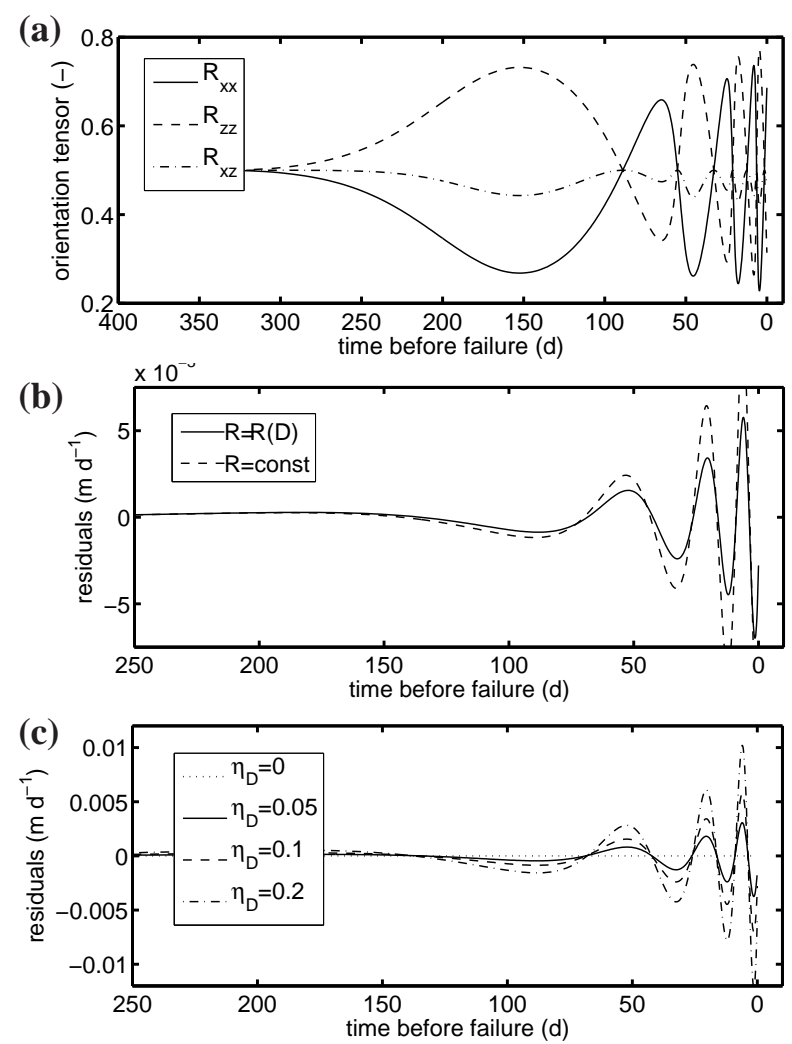

Fig. 4. Simulation of the failure of the hanging glacier with model parameters given by Table 1. (a) Evolution of the components of the damage orientation tensor $\underline{R}=\boldsymbol{v}^{(1)} \otimes \boldsymbol{v}^{(1)}$. Note that $R_{y y}=0$ since $\gamma=1$. (b) Residuals of the surface velocities fitted with Eq. (33). The surface velocities are calculated with $\underline{R}=\underline{R}(\underline{D})=\boldsymbol{v}^{(1)} \otimes \boldsymbol{v}^{(1)}$ and $\underline{R}=$ const $=\left.v^{(1)} \otimes v^{(1)}\right|_{D=\underline{0}}$. (c) Residuals of the surface velocities fitted with Eq. (33). The surface velocities are calculated with different values of $\eta_{D}$ (normed here with $\sigma_{x z}(z=0)$ ).

where

$v_{x 0}=1$,

$a_{\Omega}=2 A \Delta H\left(\left(1-\frac{\gamma}{2}\right)(k+1) B \alpha\right)^{-m_{\Omega}}$,

and

$m_{\Omega}=\frac{n}{k+1}$.

Relation (47) and differential equation (45) constitute the model describing the global acceleration of the glacier surface accompanied with oscillations.

\subsection{The homogeneous solution}

The homogeneous solution of Eq. (45) reads for $m_{\Omega} \neq 1$

$D_{x z}=C_{1} \sin \left(\frac{\left(t_{\mathrm{f}}-t\right)^{1-m_{\Omega}}}{\lambda}+\varphi\right)$, 


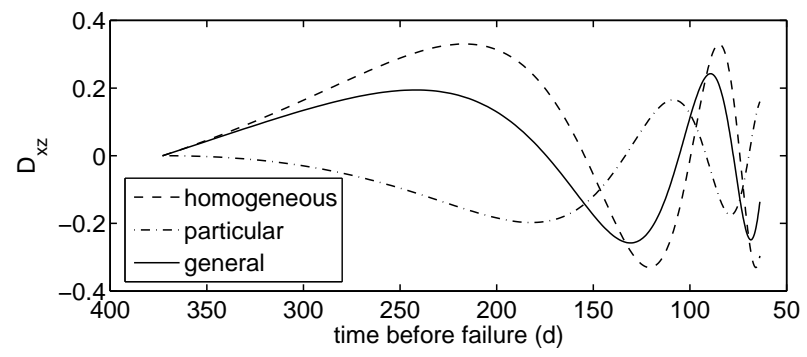

Fig. 5. Simulation of the failure of the hanging glacier with model parameters given by Table 1 . The three curves show the homogeneous, a particular and the general solution of Eq. (45) (see relation A1).

and for $m_{\Omega}=1$

$D_{x z}=C_{11} \sin \left(\frac{\ln \left(t_{\mathrm{f}}-t\right)}{\lambda_{1}}+\varphi_{1}\right)$,

where $C_{1}, \varphi, C_{11}$ and $\varphi_{1}$ are the integration constants,

$\lambda=\frac{1}{2 A}\left(m_{\Omega}-1\right)\left(\left(1-\frac{\gamma}{2}\right)(k+1) B \alpha\right)^{m_{\Omega}}$,

and

$\lambda_{1}=\frac{1}{2 A}\left(\left(1-\frac{\gamma}{2}\right)(k+1) B \alpha\right)$.

By inserting Eqs. (51) and (52) in the surface velocity (47), one obtains for $m_{\Omega} \neq 1$

$v=v_{x 0}+a_{\Omega}\left(t_{\mathrm{f}}-t\right)^{-m_{\Omega}}\left[1+C_{2} \sin \left(\frac{\left(t_{\mathrm{f}}-t\right)^{1-m_{\Omega}}}{\lambda}+\varphi\right)\right]$,

and for $m_{\Omega}=1$

$v=v_{x 0}+a_{\Omega}\left(t_{\mathrm{f}}-t\right)^{-1}\left[1+C_{21} \sin \left(\frac{\ln \left(t_{\mathrm{f}}-t\right)}{\lambda_{1}}+\varphi_{1}\right)\right]$,

where

$C_{2}=C_{1} n \eta_{D}$ and $C_{21}=C_{11} n \eta_{D}$.

Equation (55) presents a power-law acceleration with sinusoidal oscillations and amplitude variation. The oscillations have a power-law periodicity with exponent $1-m_{\Omega}$. The power periodicity reduces to a log-periodicity for $m_{\Omega}=1$ (Eq. 56). The structure of Eq. (56) is however different from the model by Sornette and Sammis (1995), since the exponent of the global power-law acceleration (in the term $\left.a_{\Omega}\left(t_{\mathrm{f}}-t\right)^{-1}\right)$ is here fixed to -1 . In the model by Sornette and Sammis it is let free (see relation 59). Note that the oscillations are possible for isotropic damage evolution $(\gamma=0)$, but only appear if the initial conditions for $D$ are non zero and anisotropic (i.e., for $C_{1} \neq 0$ or $C_{11} \neq 0$ ).

The general solution to the differential equation (45) is presented in Appendix A. Figure 5 shows the homogeneous and the general solution for the example of Sect. 4.2. In the following section, we refrain for simplicity from fitting the measurement data with the general solution of the differential equation. We assume that the homogeneous solution captures the main feature of the power-periodic oscillations.

\section{Fits of measurements}

The motion of several unstable ice masses was monitored by the Laboratory of Hydraulics, Hydrology and Glaciology (VAW) of the Swiss Federal Institute of Technology Zürich (ETHZ) within the scope of hazard assessment or research programmes. Of the various data sets collected, two will be considered here. The others do not contain enough measurements or are affected by a scattering that is too broad to be useful.

The first data set was collected at the Weisshorn east face in the Valais Alps (Switzerland). In Winter 2004-2005, an unstable ice chunk of half a million cubic meters was observed and gave cause for alarm ${ }^{10}$. In order to estimate its failure time, measurements of the unstable ice mass were performed. A theodolite laser-distometer (TPS) installed at a fixed position in front of the glacier and four reflectors mounted on stakes drilled in the unstable ice mass were used to survey the movement of the glacier. Reference reflectors, installed on a rock face close to the unstable ice mass, allowed to correct the influence of the meteorological conditions on the measurements. These corrections reduce the measurement errors to approximatively one centimeter (see Appendix B for more details). The glacier matches the hypotheses implemented above ${ }^{11}$. The surface velocity of Eq. (55) is integrated to fit the data, which are recorded as a displacement time series. For $m_{\Omega} \neq 1$ the equation of motion is 12

$$
\begin{aligned}
s(t)= & s_{0}+v_{x 0} t+\frac{a_{\Omega}}{m_{\Omega}-1}\left[\left(t_{\mathrm{f}}-t\right)^{1-m_{\Omega}}\right. \\
& \left.-C_{2} \lambda \cos \left(\frac{\left(t_{\mathrm{f}}-t\right)^{1-m_{\Omega}}}{\lambda}+\varphi\right)\right] .
\end{aligned}
$$

The equation describing log-periodic oscillation with a free exponent for the power-law acceleration (Sornette and Sammis, 1995) is also considered for comparison. It reads

$$
\begin{aligned}
s(t)= & s_{0}^{*}+v_{x 0} t+\frac{a_{\Omega}^{*}}{m_{\Omega}^{*}-1}\left(t_{\mathrm{f}}^{*}-t\right)^{1-m_{\Omega}^{*}} \\
& {\left[1-C_{2}^{*} \lambda^{*} \cos \left(\frac{\log \left(t_{\mathrm{f}}^{*}-t\right)}{\lambda^{*}}+\varphi^{*}\right)\right], }
\end{aligned}
$$

with $t_{\mathrm{f}}^{*}, m_{\Omega}^{*} \neq 1, a_{\Omega}^{*}, s_{0}^{*}, C_{2}^{*}, \lambda^{*}, \varphi^{*}$ as parameters. Note that $v_{x 0}$ is assumed to equal the parameter $v_{x 0}$ of Eq. (58).

\footnotetext{
${ }^{10}$ This unstable ice mass grew up due to in-situ snowfalls at the same location as the 1973 broken-off glacier (see Fig. 2).

${ }^{11}$ These hypotheses imply that the process of fracture is a socalled slab fracture (Pralong and Funk, 2006).

${ }^{12}$ The parameters in the next two equations are dimensional.
} 
Table 2. Values of the estimated parameters for the three data sets analyzed in this paper. The parameters are estimated using a nonlinear least squares fit. The root-mean-square error (rmse) of the fit as well as the $\chi^{2}$ statistics and its associated $p$-value are also reported. pp stands for power-periodic and $1 p$ for log-periodic oscillation functions ( 58 and 59 , respectively).

\begin{tabular}{llllllll}
\hline Parameter & Units & $\begin{array}{l}\text { Weisshorn (pp) } \\
\text { Fig. 6a }\end{array}$ & $\begin{array}{l}\text { Weisshorn (lp) } \\
\text { Fig. 6b }\end{array}$ & $\begin{array}{l}\text { Weisshorn (pp) } \\
\text { Fig. 6c }\end{array}$ & $\begin{array}{l}\text { Weisshorn (lp) } \\
\text { Fig. 6d }\end{array}$ & $\begin{array}{l}\text { Mönch (pp) } \\
\text { Fig. 7a }\end{array}$ & $\begin{array}{l}\text { Mönch (lp) } \\
\text { Fig. 7b }\end{array}$ \\
\hline$t_{\mathrm{f}}$ & $\mathrm{d}$ & 26.57 & 26.90 & 34.84 & 41.52 & 101.85 & 109.73 \\
$m_{\Omega}$ & - & 0.953 & 1.036 & 0.907 & 1.530 & 0.549 & 0.193 \\
$a_{\Omega}$ & $\mathrm{m} \mathrm{d}^{-\mathrm{m}_{\Omega}}$ & 0.789 & 0.980 & 1.937 & 18.808 & 8.171 & 42.398 \\
$s_{0}$ & $\mathrm{~m}$ & 19.54 & -24.29 & 28.39 & -5.18 & -2.30 & -1.21 \\
$v_{x 0}$ & $\mathrm{~m} \mathrm{~d}-1$ & 0.17 & 0.17 & 0.12 & 0.12 & 0.098 & 0.098 \\
$C_{2}$ & - & 0.228 & 0.001 & 0.088 & 0.005 & 0.175 & 0.021 \\
$\lambda$ & $\mathrm{d}$ & 0.022 & 2.49 & 0.028 & 0.657 & 0.011 & 0.647 \\
$\varphi$ & - & 5.82 & 3.38 & 5.38 & 0.26 & 6.06 & 6.20 \\
$\mathrm{rmse}$ & $\mathrm{m}$ & 0.0083 & 0.0083 & 0.0087 & 0.0068 & 0.0134 & 0.0121 \\
$\chi^{2}$ & - & 1.1 & 1.1 & 1.2 & 0.8 & 7.7 & 7.1 \\
$p$ & - & 0.89 & 0.89 & 0.88 & 0.93 & 0.10 & 0.13 \\
\hline
\end{tabular}

Figure 6 shows the motion of two reflectors ${ }^{13}$ and their fits performed with Eqs. (58) and (59). Although the time of failure has been registered, we leave it an unknown parameter here. Fitted values of the parameters of Eqs. (58) and (59) are listed in Table 2. All fits match the measurements well (see also root mean square error and $\chi^{2}$-test of the fit in Table 2).

The second data set describes the motion of a stake with reflector installed on an unstable ice mass at the front of the Mönch hanging glacier, Switzerland (Pralong et al., 2005) ${ }^{14}$. The measurements were performed with the same equipment as for the Weisshorn hanging glacier. The time of failure of the unstable mass is not known as a subfailure occurred prior to the main failure, and resulted in the loss of the measurement equipment on the glacier. This glacier does not match hypothesis expressed in Eq. (13) since the geometry of the unstable ice mass is not shallow ${ }^{15}$. Despite this violation, we fit the data with the power-periodic function, and compare it to the fit performed with the log-periodic function (Fig. 7). Parameters values are listed in Table 2. Both fits match the measurements well (see also root mean square error and $\chi^{2}$ test in Table 2).

For the derivation of the analytical solution, we assumed that the time of failure occurs at infinite velocity (relation 37). According to this assumption, the power-periodic oscillations predict the time of failure more accurately. However

\footnotetext{
${ }^{13}$ We refrain to present the fits of the four points, since all show similar behaviors.

${ }^{14}$ The motion of three points was actually recorded during this measurement campaign. All three give similar results. For more details about the data set and the reconstruction of the displacement of the reflectors from the measurements, see the reference.

${ }^{15}$ The fracture at the front of the glacier occurs according to a so-called wedge fracture (Pralong and Funk, 2006).
}

this assumption is not physical, since a transition from subcritical to critical fracture exists (e.g., Lemaitre, 1996). The subcritical fracture is described by the proposed model (relation 55). In comparison to the duration of the subcritical fracture (order of magnitude of a month), the critical fracture can be considered as instantaneous. Therefore the transition from subcritical to critical fracture defines the time of failure. Since the criterion for this transition is not known (Sect. 4.2), the time lag observed between the observed and the predicted time of failure (especially in the case of the log-periodic model) can be related to the inaccuracy of assumption (37).

\section{Discussion}

The distinction between log- and power-periodic oscillations is difficult to identify for natural processes: First, the amplitude of the oscillations is small compared to the accuracy of the measurements. Second, the lacking data in the time series makes the fitting hazardous, particularly for fitting oscillations. Third, the result of the fit for accelerating oscillations depends on the value of the constant velocity $v_{x 0}$. The identification of this parameter is subject to uncertainties, since it is strongly correlated with the other parameters of Eqs. (58) or (59). Fourth, measurements conducted close to the failure time are difficult to perform (at least on hanging glaciers: loss of equipment in fresh crevasses, sub-failures). Moreover, measurements can realistically only be started when clear signs of destabilization are observed. It follows that the magnitude of the time window, when measurements are performed, is often too small to allow accurate identification of the parameters (Pralong et al., 2006) and the two oscillation models to be clearly differentiated. The measurements 
(a)
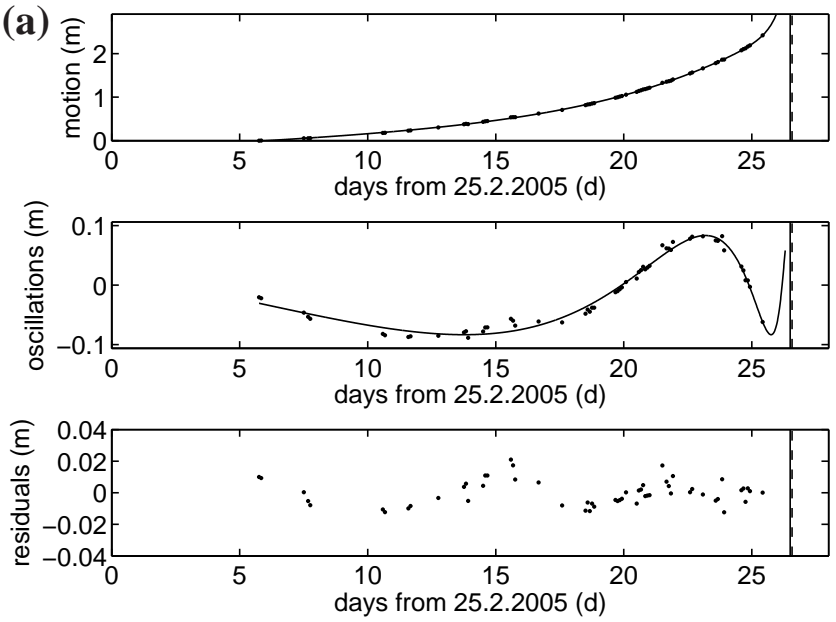

(c)
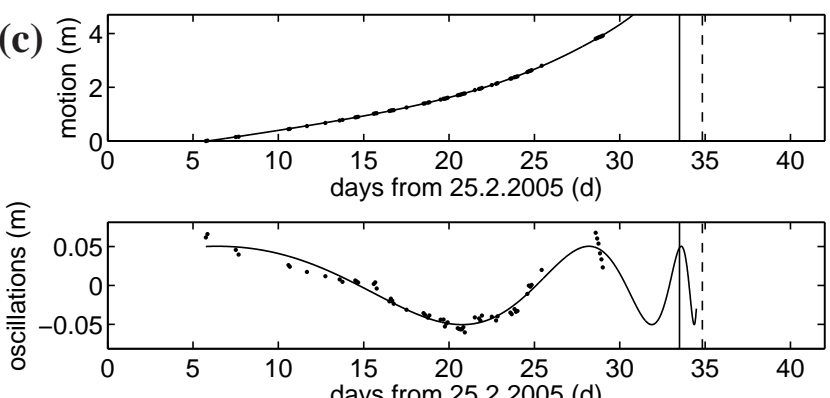

days from 25.2.2005 (d)

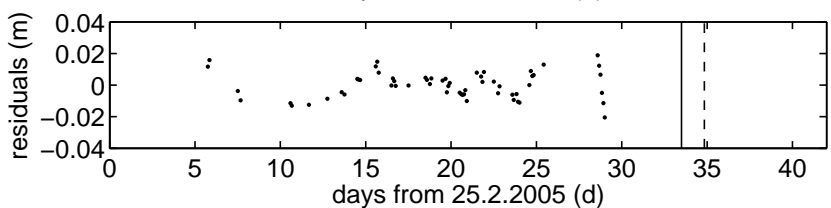

(b)
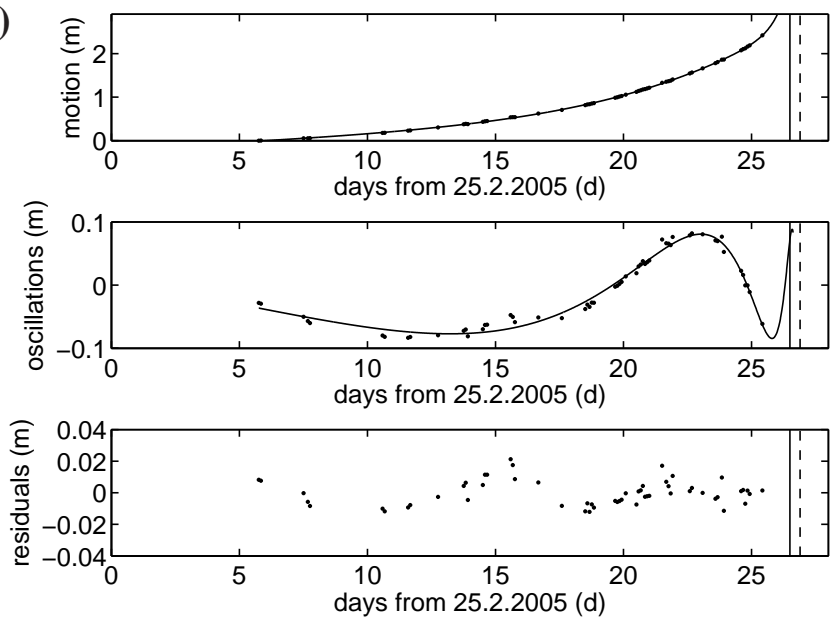

(d)
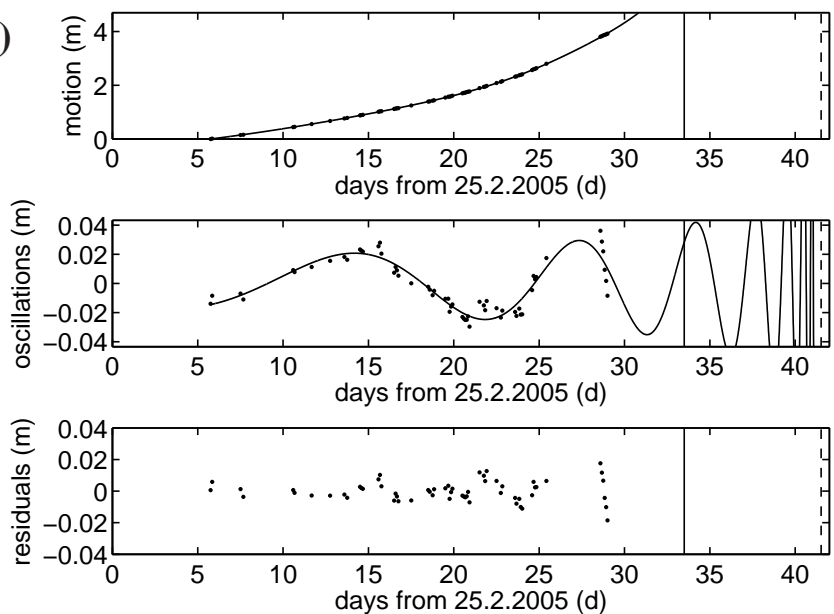

Fig. 6. Fit of the data set of the Weisshorn glacier. Each panel contains the data with its fit (the data and the fit are plotted without their constant velocity component $v_{0} t$ ), the zoom on the oscillation with its fit, and the residuals. (a) Surface displacement of the first point fitted with the power-periodic oscillation function (58). (b) Surface displacement of the first point fitted with the log-periodic oscillation function (59). The unstable ice mass where this point was located broke off 26.5 days after the beginning of the measurements (vertical solid lines). The estimated failure time (estimated with the fit) is reported with vertical dashed lines (see also Table 2). (c) Surface displacement of the second point fitted with the power-periodic oscillation function (58). (d) Surface displacement of the second point fitted with the log-periodic oscillation function (59). The unstable ice mass where this point was located broke off 33.5 days after the beginning of the measurements (vertical solid lines). The estimated failure time is reported with vertical dashed lines (see also Table 2). The lacks in the time series correspond to periods of cloudy weather.

performed at the Weisshorn suggest however that the powerperiodic and the log-periodic oscillations become similar for this failure event, since $m_{\Omega}$ and $m_{\Omega}^{*}$ are close to one. This is not the case for the measurements at the Mönch where $m_{\Omega} \neq 1$ and $m_{\Omega}^{*} \neq 1$, possibly because this glacier violates the geometrical assumption (13).

The power-periodic oscillations result from the combination of damage anisotropy and ice rotation due to shearing deformation. The global acceleration of the glacier due to damage accumulation induces an increase of the vorticity rate in the basal damage layer subject to shearing. The dam- age is advected by the ice motion and rotates according to the rotation of the ice. The values of the components of the damage tensor, describing (in a fix reference) the anisotropy of the rotating damage, oscillate with a frequency proportional to the vorticity rate of the ice which shows a power-law acceleration. These components influence the ice flow and induce the power-periodic oscillations.

More precisely, in the equations of the model, the value $D=\frac{D_{x x}+D_{z z}}{2}$ drives the global power-law acceleration (relations 28 with 25). $D$ does not depend on the ice rotation, i.e., it does not oscillate with time (Fig. 3c) since $D$ 

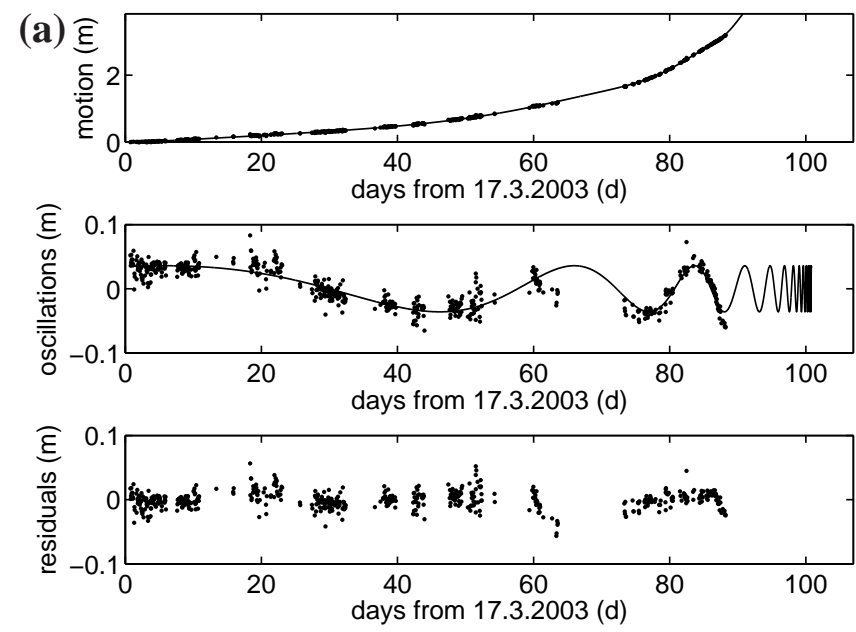

(b)
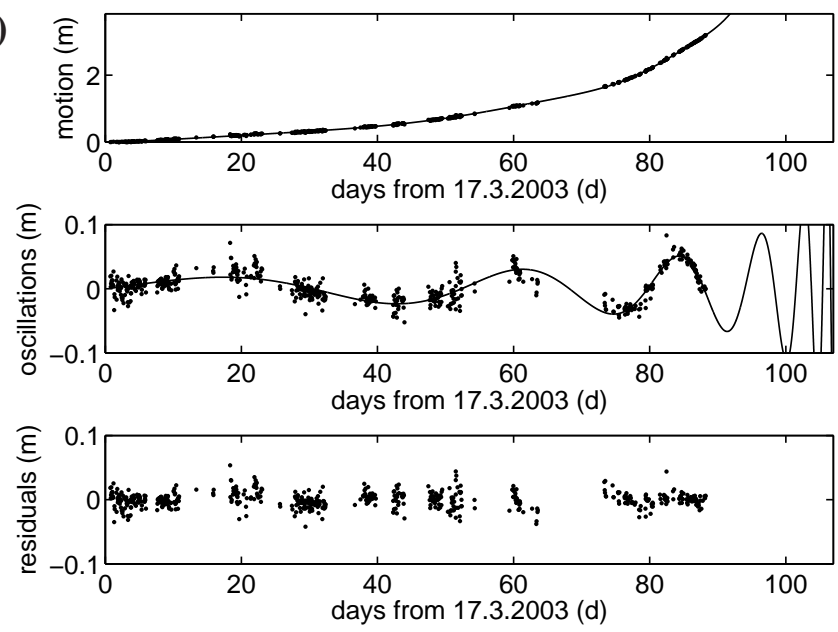

Fig. 7. Fit of the data set of the Mönch glacier. Each panel contains the data with the fit (the data and the fit are plotted without their constant velocity component $v_{0} t$ ), the zoom on the oscillation with its fit, and the residuals. (a) Surface displacement fitted with the powerperiodic oscillation function (58). (b) Surface displacement fitted with the log-periodic oscillation function (59). The lacks in the time series correspond to periods of cloudy weather.

is proportional to the first invariant of $\underline{D}$. The oscillations accompanying the global power-law acceleration result from the oscillations of the component $D_{x z}$ (Fig. 3c) of the damage tensor $\underline{D}$ since $D_{x z}$ influences the ice flow according to Eq. (47). The oscillations of $D_{x z}$ result from its dynamic interaction with the damage components $D^{-}$described in the two first order $\operatorname{ODE}\left(43_{c}\right)$ and $\left(43_{d}\right)^{16}$. The interacting terms, leading to the oscillation of $D^{-}$and $D_{x z}$, emerge in these ODE because of the use of the Jaumann derivative to describe the rotation of the anisotropic damage $\underline{D}$ (relation 3). Therefore the oscillations of $D_{x z}$ and $D^{-}$reflect the rotation of the damage advected by the ice. The oscillations accelerate since the term $(1-D)^{-n}$ in Eqs. (43) grows as a power-law function. This growth corresponds to the increase of the vorticity rate of the spin tensor $\underline{W}$, which describes the ice rotation. Therefore $D_{x z}$ and $D^{-}$show power-periodic oscillations.

Accelerating oscillations emerge in systems having a positive feedback (which leads to finite time singularities, e.g., creep rupture), coupled to a stable second order dynamics (which leads to oscillations, e.g., strain depending healing, harmonic oscillator) (e.g., Ide and Sornette, 2002). In this model the positive feedback is given by the accumulation of damage $D$. The stable second order dynamics is described by the second order ODE for $D_{x z}$ (relation 45), which results from the two coupled first order ODE (relations $43_{c}$ and $43_{d}$ ) for $D^{-}$and $D_{x z}$.

\footnotetext{
${ }^{16}$ The variables $D, D_{y y}, D^{-}$and $D_{x z}$ in Eq. (43) can be interpreted as follows: $D$ is the mean isotropic damage which is independent of ice rotation. $D_{y y}$ is the damage in the y-direction and does not interact with the other variables. $D^{-}$and $D_{x z}$ are pure anisotropic damage components (without the influence of the mean isotropic damage $D$ ) in two different anisotropic directions.
}

\section{Conclusions}

Power-periodic oscillations have been derived from the theory of the continuum damage mechanics. The components of the damage tensor have been first shown to oscillate with a power-periodic frequency. These oscillations are caused by the anisotropy of the damage coupled to the rotation of the ice at the glacier base. The rotation of the ice is caused by the shearing deformation due to ice flow. The oscillation of the components of the damage tensor have been then shown to be transmitted to the glacier surface velocity. This transfer results in power-periodic oscillations accompanying the global power-law acceleration of the glacier.

The power-periodic oscillation model adequately fits the oscillations which are observed to accompany the global power-law acceleration of glaciers. Therefore the proposed model provides an alternative approach to the classical logperiodic model (where the oscillations emerge because of discrete scale invariance properties) to explain accelerating oscillations for critical shearing. Since differences with logperiodic oscillations have been shown to emerge close to the failure time, comparison between both types of oscillations should be performed on time series covering more than one order of magnitude (which is not the case here).

The power-periodic oscillations generalize the logperiodic ones, since the power-periodicity converges to the log-periodicity when the exponent of the power-periodicity equals one. In this study, the exponent has been identified to be close to one only for the Weisshorn glacier, which matches all model assumptions. Further validations should be performed on that type of glaciers to establish whether this similarity can be retrieved. 
The power-periodic oscillation model can be applied to other localized shearing fractures occurring in viscous media.

\section{Appendix A}

\section{General solution of the analytical problem}

The general solution to Eq. (45) reads for $m_{\Omega} \neq 1$

$$
\begin{aligned}
D_{x z}= & C_{1} \sin \left(\frac{\left(t_{\mathrm{f}}-t\right)^{1-m_{\Omega}}}{\lambda}+\varphi\right) \\
+ & C_{3} \int_{0}^{t} \sin \left(\frac{\left(t_{\mathrm{f}}-\theta\right)^{1-m_{\Omega}}}{\lambda}\right)\left(t_{\mathrm{f}}-\theta\right)^{m_{\Omega}-k_{\Omega}-1} d \theta \\
& \cos \left(\frac{\left(t_{\mathrm{f}}-t\right)^{1-m_{\Omega}}}{\lambda}\right) \\
- & C_{3} \int_{0}^{t} \cos \left(\frac{\left(t_{\mathrm{f}}-\theta\right)^{1-m_{\Omega}}}{\lambda}\right)\left(t_{\mathrm{f}}-\theta\right)^{m_{\Omega}-k_{\Omega}-1} d \theta \\
& \sin \left(\frac{\left(t_{\mathrm{f}}-t\right)^{1-m_{\Omega}}}{\lambda}\right),
\end{aligned}
$$

and for $m_{\Omega}=1$

$D_{x z}=C_{11} \sin \left(\frac{\ln \left(t_{\mathrm{f}}-t\right)}{\lambda_{1}}+\varphi_{1}\right)-C_{31}\left(t_{\mathrm{f}}-t\right)^{\frac{1}{k+1}}$,

where $C_{1}, \varphi, C_{11}$ and $\varphi_{1}$ are the integration constants and

$\lambda=\frac{1}{2 A}\left(m_{\Omega}-1\right)\left(\left(1-\frac{\gamma}{2}\right)(k+1) B \alpha\right)^{-m_{\Omega}}$,

$\lambda_{1}=\frac{1}{2 A}\left(\left(1-\frac{\gamma}{2}\right)(k+1) B \alpha\right)$,

$m_{\Omega}=\frac{n}{k+1}$,

$k_{\Omega}=\frac{k}{k+1}$,

$C_{3}=\frac{\gamma}{4} \frac{B}{A} \alpha\left(k_{\Omega}-m_{\Omega}\right)\left[\left(1-\frac{\gamma}{2}\right)(k+1) B \alpha\right]^{m_{\Omega}-k_{\Omega}}$,

$C_{31}=\frac{\gamma}{8} \frac{B}{A^{2}} \alpha(k+1)\left(2 A \lambda_{1}\right)^{2-k_{\Omega}}\left[(k+1)^{2}+\lambda_{1}^{2}\right]^{-1}$.

\section{Appendix B}

\section{Reconstruction of the displacement of reflectors measured on the Weisshorn hanging glacier}

The hanging glacier at the Weisshorn is located at about $4200 \mathrm{~m}$ a.s.l. For convenience, the laser-distometer used to measure the displacement of the reflectors on the glacier was installed at a distance of about $6 \mathrm{~km}$ from the glacier, approximatively in the direction of the glacier flow. At such a distance, the accuracy of the measurements (corrected with reference reflectors located on rocks close to the glacier) is less than $1 \mathrm{~cm}$ in the direction of the measurement (which corresponds to the accuracy of the measurement of the distance) and about $50 \mathrm{~cm}$ in the perpendicular direction (corresponding to the accuracy of the measurement of the azimuth or elevation angle times the distance). As a result, the position, i.e. the displacement of the reflectors calculated with the distance and the angles is not accurate. This result can however be used to estimate the angle between the direction of measurement and the flow direction, since it furnish a three dimensional representation of the motion of the reflectors. The displacement of the reflectors in the flow direction (which corresponds to the data set presented here) is then calculated with the measurement of the distance corrected with the angle between the direction of measurement and the flow direction.

Acknowledgements. The author wishes to thank D. Amitrano, K. Hutter, D. Sornette and M. Funk for valuable comments.

Edited by: G. Zoeller

Reviewed by: D. Amitrano and another referee

\section{References}

Glen, J. W.: Experiments on the deformation of ice, J. Glaciol., 2, 111-114, 1952.

Ide, K. and Sornette, D.: Oscillatory finite-time singularities in finance, population and rupture, Physica A-Statistical Mechanics and its Applications, 307, 63-106, 2002.

Kachanov, L. M.: Rupture time under creep conditions (trans. from Russian, 1957), Int. J. Fract., 97, xi-xviii, 1999.

Lemaitre, J.: A course on damage mechanics, Springer, Berlin, Germany, second edn., 228 p., 1996.

Murakami, S. and Ohno, N.: A continuum theory of creep and creep damage, in: Proc. of the 3rd IUTAM Symposium on Creep in Structures, Leicaster, UK, edited by: Ponter, A. R. S. and Hayhurst, D. R., pp. 422-444, 1980.

Paterson, W. S. B.: The Physics of Glaciers, Pergamon, New York, third edn., 1994.

Pralong, A. and Funk, M.: Dynamic Damage Model of Crevasse Opening and Application to Glacier Calving, J. Geophys. Res., 110, B01309, doi:10.1029/2004JB003104, 2005.

Pralong, A. and Funk, M.: On the instability of avalanching glaciers, J. Glaciol., 52, 31-48, 2006.

Pralong, A., Birrer, C., Stahel, W., and Funk, M.: On the Predictability of Ice Avalanches, Nonlin. Processes Geophys., 12, 849-861, 2005, http://www.nonlin-processes-geophys.net/12/849/2005/.

Pralong, A., Hutter, K., and Funk, M.: Anisotropic Damage Mechanics for Viscoelastic Ice, Continuum Mechanics and Thermodynamics, 17, 387-408, doi:10.1007/s00161-005-0002-5, 2006.

Rabotnov, Y. N.: Creep problems in structural members, NorthHolland, 822 p., 1969.

Röthlisberger, H.: Eislawinen und Ausbrüche von Gletscherseen, in: Gletscher und Klima - glaciers et climat, edited by: Kasser, P., Jahrbuch der Schweizerischen Naturforschenden Gesellschaft, wissenschaftlicher Teil 1978, pp. 170-212, Birkhäuser Verlag Basel, Boston, Stuttgart, 1981. 
Sahimi, M. and Arbabi, S.: Scaling Laws for Fracture of Heterogeneous Materials and Rock, Phys. Rev. Lett., 77, 3689-3692, 1996.

Sornette, D.: Discrete-scale invariance and complex dimensions, Physics Reports - Review section of physics letters, 297, 239270, 1998.

Sornette, D. and Sammis, C. G.: Complex critical exponents from renormalization-group theory of earthquakes - implications for earthquake predictions, J. Phys. I, 5, 607-619, 1995.
Voight, B.: A method for prediction of volcanic eruptions, Nature, 332, 125-130, 1988.

Zhou, W. X. and Sornette, D.: Generalized q analysis of logperiodicity: Applications to critical ruptures, Phys. Rev. E, 66, 046111, Part 2, 2002. 